\section{LJ000328, a novel ALK2/3 kinase inhibitor, represses hepcidin and significantly improves the phenotype of IRIDA}

Matriptase-2 (MT2), a transmembrane serine protease expressed mainly in the liver, plays an essential role in the regulation of iron homeostasis. Mutations in the MT2-encoding gene (TMPRSS6) result in excessive hepcidin expression and iron-refractory iron-deficiency anemia (IRIDA) in both humans and mice. ${ }^{1,2}$ IRIDA is an autosomal recessive disorder characterized by congenital hypochromic, microcytic anemia, low mean corpuscular erythrocyte volume, low transferrin saturation, and defects in iron absorption and utilization. ${ }^{3}$ TMPRSS6 mutations affect around 100 patients worldwide, although probably underestimated, who are unresponsive to oral iron treatment and only partially responsive to parenteral iron therapy. Therefore, there is an urgent need for novel therapeutic approaches for the management of IRIDA. Hepcidin expression is physiologically regulated by the body iron status via the BMP-SMAD signaling pathway that is initiated by the binding of BMP2 ${ }^{4}$ and $\mathrm{BMP}^{5}$ to transmembrane serine-threonine kinases, the BMP type $\mathrm{I}^{6}$ (particularly ALK2 and ALK3) and type $\mathrm{II}^{7}$ receptors. This results in the phosphorylation of the SMAD 1/5/8 effectors, translocation of the phosphorylated SMAD bound to SMAD4 to the nucleus, and upregulation of hepcidin gene transcription. ${ }^{8} \mathrm{MT} 2$ is an inhibitor of this pathway and functional loss of MT2 leads to inappropriate activation of BMP signaling and elevated hepcidin levels. Increased hepcidin downregulates the iron exporter ferroportin ${ }^{9}$ and thereby decreases the amount of iron released from the enterocytes and the body iron stores to the blood compartment. Impairment of dietary iron absorption results in hypoferremia and microcytic anemia. Interestingly, deletion of $B m p 6$ in Tmprss $6^{-/}$mice reduced their excessive hepcidin production and correct-
A<smiles>Cc1cc(-c2cnc3cc(-c4ccc(N5CCNCC5)cc4)ccn23)c2cc(F)ccc2n1</smiles>

6-fluoro-2-methyl-4-(7-(4-(piperazin-1yl)phenyl)imidazo[1,2-a]pyridin-3yl)quinoline
B

\begin{tabular}{|c|c|}
\hline BMP Type-I receptors & IC $_{\mathbf{5 0}}$ (nM) \\
\hline ALK1 & 166.3 \\
\hline ALK2 & 10.9 \\
\hline ALK3 & 5.1 \\
\hline ALK6 & 29.4 \\
\hline \multicolumn{2}{|c|}{} \\
\hline TGF $\beta$ Type-I receptors \\
\hline ALK4 & 568.7 \\
\hline ALK5 & 1267 \\
\hline \multicolumn{2}{|c|}{} \\
\hline Type-II receptors & $>50000$ \\
\hline BMPR2 & 587.2 \\
\hline TGFBR2 &
\end{tabular}

D

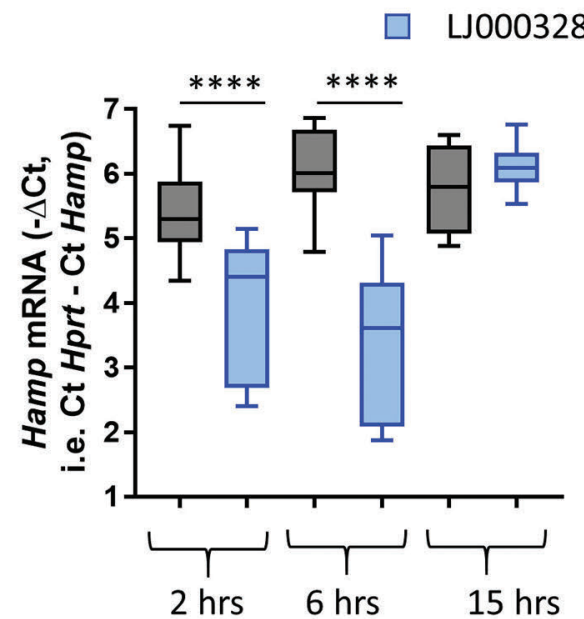

Figure 1. $\mathrm{L} 000328$ is selective of the BMP Type I receptors and is a potent inhibitor of liver hepcidin expression. (A) U000328 chemical structure. (B) Selectivity of $\mathrm{L} 000328$ for different BMP, TGF- $\beta$, and activin receptors, determined by in vitro kinase assays. Wild-type (WT) C57BL/ 6 male mice injected with ப000328 (blue boxes) (8-10/group) were sacrificed 2, 6, and 15 hours after injection and compared to mock-injected mice (grey boxes). Mice were analyzed for liver (C) P-SMAD5 relative to total SMAD5 protein expression and (D) Hamp mRNA expression. Box-and-whisker plots are shown for Hamp $\triangle$ Ct values. Mean $\Delta$ Ct values were compared by a Student's t-test. ****P<0.0001. 
ed the microcytic anemia, ${ }^{10}$ suggesting that targeting $\mathrm{BMP}-\mathrm{SMAD}$ signaling could offer an effective therapeutic approach to the correction of anemia in IRIDA patients. In this study, to improve the iron deficiency and the microcytic anemia caused by the deletion of MT2, we inhibited the BMP-SMAD signaling with a novel ALK2/3 inhibitor, LJ000328. We demonstrate that only a few weeks of treatment are sufficient to strongly repress hepcidin expression and significantly improve the microcytic anemia in Tmprss6 $6^{-/}$mice.

Dorsomorphin $^{11}$ and LDN-193189, ${ }^{12}$ two small molecule repressors of hepcidin expression, are potent inhibitors of the kinase activity of BMP type I receptors, but they also exhibit significant TGF- $\beta$ receptor inhibition
A

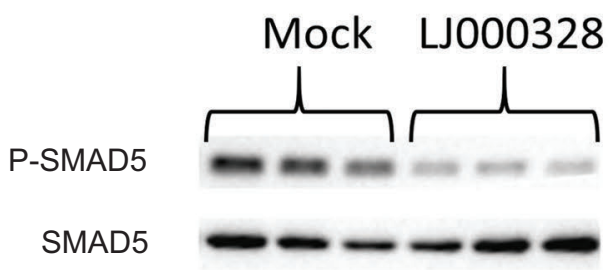

B

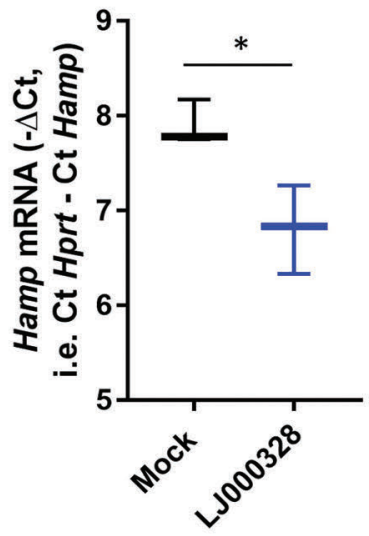

C

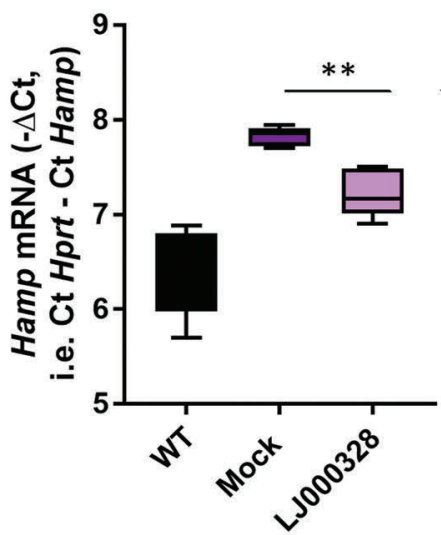

$\mathbf{F}$

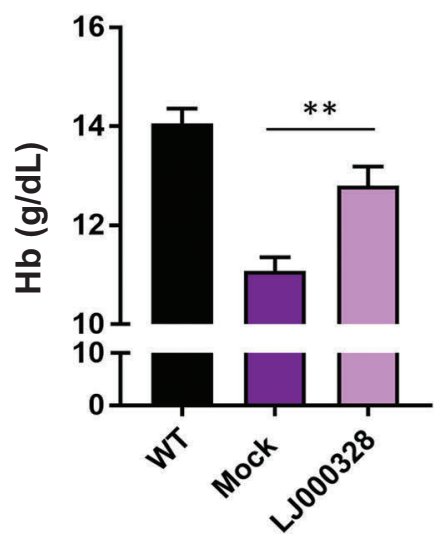

D

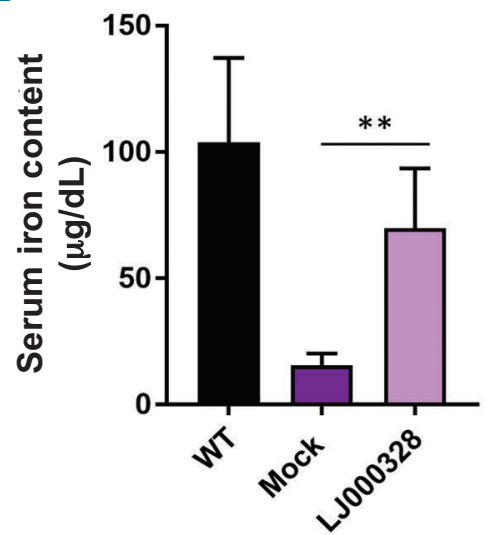

G

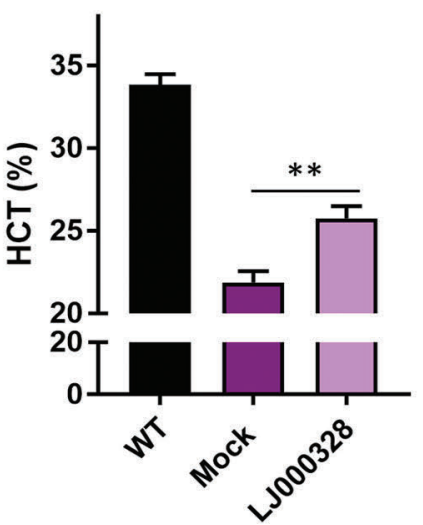

$\mathbf{E}$

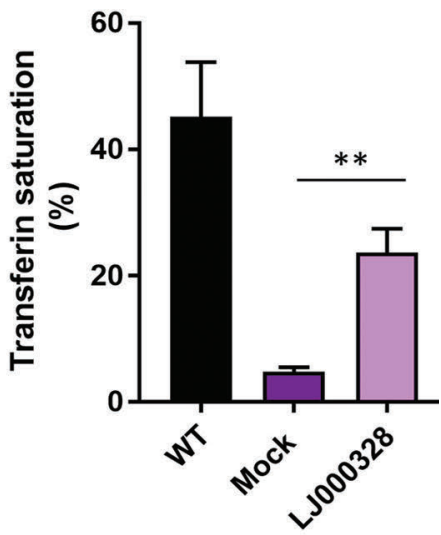

H

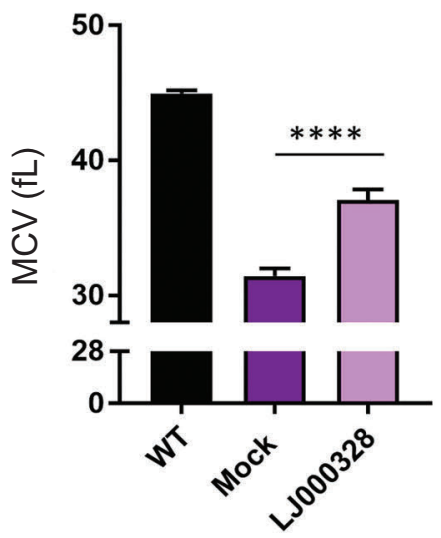

Figure 2. L000328 inhibits hepcidin expression and improves the anemia of Tmprss6 mice. Tmprss6 male mice injected with L000328 (blue boxes) ( $\mathrm{n}=3$ ) were sacrificed 2 hours after injection and compared to mock-injected mice (grey boxes) ( $n=4)$. Mice were analyzed for liver (A) P-SMAD5 relative to total SMAD5 protein expression and (B) Hamp mRNA expression. Tmprss6 male mice were injected with L000328 (pink boxes) ( $\mathrm{n=5}$ ) for seven weeks and compared with mock-injected mice (purple boxes) $(n=5)$. Mice were analyzed for $(C)$ liver Hamp mRNA expression, (D) serum iron content, (E) transferrin saturation, $(F)$ hemoglobin, $(\mathrm{G})$ hematocrit, and $(\mathrm{H})$ mean corpuscular volume. For comparison, black boxes indicate levels measured in wild-type (WT) mice. Means \pm standard error of the mean (SEM) are shown for hematological parameters, and box-and-whisker plots for Hamp $\Delta$ Ct values. Mean comparisons were made by Student's $t$-tests. $* * P<0.01$. 
at moderate concentrations. ${ }^{13}$ Thus, while dorsomorphin and LDN-193189 are valuable tool compounds, they lack sufficient target specificity for clinical testing, particularly for indications requiring long-term treatment. LJ00328 (Figure 1A) is a member of a new class of BMP type I receptor inhibitors with improved in vivo pharmacodynamics. Moreover, LJ000328 exhibits substantially greater selectivity for BMP than for TGF- $\beta$ and activin type I receptors, as shown in in vitro kinase assays (Figure 1B and Online Supplementary Table S2). Indeed, LJ000328 demonstrated the greatest potency against ALK3 and ALK2, which are the BMP type 1 receptors whose deletion in hepatocytes induces hepcidin repression in vivo. ${ }^{6}$ These data suggest that LJ000328 might be efficient to reduce hepcidin in vivo.

To investigate this possibility further, C57BL/6J male wild-type mice received a single intraperitoneal injection of LJ000328 $(20 \mathrm{mg} / \mathrm{kg}$; diluted in 2-hydroxypropyl- $\beta$ cyclodextrin solution) and were sacrificed 2,6 , or 15 hours later. Interestingly, LJ000328 injection inhibited BMP-SMAD signaling in the liver for up to 6 hours, as illustrated by a decrease in Smad5 phosphorylation (Figure 1C) and Id1 and Smad7 levels (Online Supplementary Figure $S 1 A-B)$. As a consequence, hepcidin was reduced by $83.3 \%$ ( $95 \%$ confidence interval [CI]: 75.6-89.9) 6 hours post-LJ000328 injection (Figure 1D), leading to serum iron content and transferrin saturation increases (Online Supplementary Figure S1C-D). Of note, BMP type I and II receptor expression was not affected by the treatment (Online Supplementary Figure S2). We next assessed whether LJ000328 also inhibits hepcidin expression in Tmprss $6^{-/}$male mice, despite their excessive BMP-SMAD signaling. Promisingly, we observed a significant inhibition of Bmp signaling and hepcidin expression two hours after LJ000328 administration (Figure 2A-B and Online Supplementary Figure $S 3 A-B$ ), suggesting that prolonged LJ000328 administration could impact the excessive hepcidin production seen in the absence of MT2 and correct at least in part the anemia associated to the IRIDA mouse model.

Based on these results, LJ000328 was administered twice-daily to Tmprss6 $6^{--}$mice for several weeks. After seven weeks of treatment, the alopecia usually observed in these mice had disappeared (Online Supplementary Figure $S 4 A$ ), which can be explained by the repression of $\mathrm{BMP}$ signaling and hepcidin sustained over time (Online Supplementary Figure $S 4 B$ and Figure 2C), allowing more dietary iron to be absorbed. We indeed noticed a massive increase in serum iron content and transferrin saturation, compared with mock-injected Tmprss6 $6^{-1}$ mice (Figure 2DE). Most notably, the microcytic anemia caused by the lack of MT2 was partially corrected, leading to decreased serum erythropoietin level (Online Supplementary Figure $S 4 C$ ) and improved hematological parameters, with notably significant increases in hemoglobin, hematocrit, and mean corpuscular volume in mice that received LJ000328, compared with mock-injected mice (Figure 2F-H). Although hemoglobin did not reach the level seen in wild-type mice, the increase of $1.7 \mathrm{~g} / \mathrm{dL}$ observed here is comparable to the hemoglobin rise gained after intravenous iron therapy in children with IRIDA. ${ }^{14}$ Importantly, injections of LJ000328 were well tolerated. We observed no weight loss (Online Supplementary Figure S5A) and importantly no liver damage characterized by the measurement of serum transaminases, serum creatinine and lactate dehydrogenase (Online Supplementary Figure $S 5 B-E$ ) and no signs of inflammation and fibrosis (Online Supplementary Figure
S5F-H). Moreover, after seven weeks of treatment, no signs of peritonitis, and no histopathological abnormalities in the liver, spleen, heart and kidney were seen (Online Supplementary Figures S6-9).

Currently, IRIDA patients are treated with intravenous iron injections. However, there are safety concerns about parenteral iron therapy, as it may increase the risk of infection and favor oxidative stress. Therefore, the development of new therapies is essential. We show in the present study that the novel kinase inhibitor LJ000328 is a very promising small molecule for the treatment of IRIDA. First, it is highly selective for BMP compared with TGF- $\beta$ type I receptors, which should alleviate toxicity concerns associated with TGF- $\beta$ inhibition. Second, it strongly represses hepcidin and significantly improves the microcytic anemia after only a few weeks of treatment. Third, given its low molecular weight, it easily crosses the intestinal barrier and represses hepcidin after oral administration (Online Supplementary Figure S10). Therefore, in contrast to the similarly efficient antihemojuvelin antibody ${ }^{15}$ it will be active when administered orally, which offers patients significant advantages. Although we did not observe toxicity after seven weeks of treatment, the drug safety of LJ000328 is still under assessment to evaluate potential toxicities of prolonged $\mathrm{BMP}$ signaling inhibition in the liver.

In line with this study, the ability of LJ000328 to suppress hepcidin expression could be a novel therapeutic option for the treatment of others iron-restricted anemias caused by increased hepcidin level such as anemia of inflammation, cancer, or chronic kidney disease. Further studies will be required to address this possibility.

Audrey Belot, ${ }^{1}$ Ophélie Gourbeyre, ${ }^{1}$ Alexis Fay, ${ }^{1}$ Anais Palin, Céline Besson-Fournier, ${ }^{1}$ Chloé Latour, ${ }^{1}$ Corey R. Hopkins, ${ }^{2}$ George F. Tidmarsh, ${ }^{3}$ Hélène Coppin, ${ }^{1}$ Marie-Paule Roth, Matthew R. Ritter, ${ }^{3}$ Charles C. Hong ${ }^{4}$ and Delphine Meynard'

'IRSD, Université de Toulouse, INSERM, INRA, ENVT, UPS,

Toulouse, France; ${ }^{2}$ Department of Pharmaceutical Sciences, College of Pharmacy, University of Nebraska Medical Center, Omaha, NE,

USA; ${ }^{3}$ La Jolla Pharmaceutical Company, San Diego, CA, USA and ${ }^{4}$ Division of Cardiovascular Medicine, University of Maryland School of Medicine, Baltimore, USA

\section{Correspondence: DELPHINE MEYNARD}

delphine.meynard@inserm.fr

\section{doi:10.3324/haematol.2019.236133}

Acknowledgments: the authors are grateful to Carlos Lopez Otin (University of Oviedo, Oviedo, Spain) for kindly providing the original Tmprss6-1- mice on a mixed genetic background. They also thank members of the INSERM USO06 facility (Toulouse) for their technical assistance and help in the mouse breeding.

Funding: DM was supported by the Cooley's Anemia Foundation, the French Foundation for Rare Diseases, the Région Midi-Pyrénées and ANR (ANR-17-CE14-0036-01 and ANR-17-CE14-0031-01). $H C$ and $M-P R$ were supported in part by grants from FRM (DEQ2000326528) and ANR (ANR-13-BSV3-0015-01). This work was also supported by the "Programme des Investissements d'Avenir" ANINFIMIP (ANR-11EQPX-0003).

Information on authorship, contributions, and financial \& other disclosures was provided by the authors and is available with the online version of this article at wWw. haematologica.org.

\section{References}

1. Wang CY, Meynard D, Lin HY. The role of TMPRSS6/matriptase-2 in iron regulation and anemia. Front Pharmacol. 2014;5:114

2. De Falco L, Sanchez M, Silvestri L, et al. Iron refractory iron deficien- 
cy anemia. Haematologica. 2013;98(6):845-853

3. Finberg KE, Heeney MM, Campagna DR, et al. Mutations in TMPRSS6 cause iron-refractory iron deficiency anemia (IRIDA). Nat Genet. 2008;40(5):569-571.

4. Koch PS, Olsavszky V, Ulbrich F, et al. Angiocrine Bmp2 signaling in murine liver controls normal iron homeostasis. Blood. 2017;129(4):415-419.

5. Meynard D, Kautz L, Darnaud V, Canonne-Hergaux F, Coppin H, Roth MP. Lack of the bone morphogenetic protein BMP6 induces massive iron overload. Nat Genet. 2009;41(4):478-481.

6. Steinbicker AU, Bartnikas TB, Lohmeyer LK, et al. Perturbation of hepcidin expression by BMP type I receptor deletion induces iron overload in mice. Blood. 2011;118(15):4224-4230.

7. Mayeur C, Leyton PA, Kolodziej SA, Yu B, Bloch KD. BMP type II receptors have redundant roles in the regulation of hepatic hepcidin gene expression and iron metabolism. Blood. 2014;124(13):21162123 .

8. Truksa J, Lee P, Beutler E. Two BMP responsive elements, STAT, and bZIP/HNF4/COUP motifs of the hepcidin promoter are critical for BMP, SMAD1, and HJV responsiveness. Blood. 2009;113(3):688-695.

9. Nemeth E, Tuttle MS, Powelson J, et al. Hepcidin regulates cellular iron efflux by binding to ferroportin and inducing its internalization. Science. 2004;306(5704):2090-2093.

10. Nai A, Rubio A, Campanella A, et al. Limiting hepatic Bmp-Smad signaling by matriptase- 2 is required for erythropoietin-mediated hepcidin suppression in mice. Blood. 2016;127(19):2327-2336.

11. Yu PB, Hong CC, Sachidanandan C, et al. Dorsomorphin inhibits BMP signals required for embryogenesis and iron metabolism. Nat Chem Biol. 2008;4(1):33-41.

12. Steinbicker AU, Sachidanandan C, Vonner AJ, et al. Inhibition of bone morphogenetic protein signaling attenuates anemia associated with inflammation. Blood. 2011;117(18):4915-4923.

13. Hao J, Ho JN, Lewis JA, et al. In vivo structure-activity relationship study of dorsomorphin analogues identifies selective VEGF and BMP inhibitors. ACS Chem Biol. 2010;5(2):245-253.

14. Akin M, Atay E, Oztekin $O$, et al. Responsiveness to parenteral iron therapy in children with oral iron-refractory iron-deficiency anemia. Pediatr Hematol Oncol. 2014;31(1):57-61.

15. Kovac S, Boser P, Cui Y, et al. Anti-hemojuvelin antibody corrects anemia caused by inappropriately high hepcidin levels. Haematologica. 2016;101(5):e173-176. 